\title{
Proposta e avaliação de algoritmo de médias sucessivas para reconstrução da matriz origem-destino sintética
}

\author{
Bruno Vieira Bertoncini ${ }^{1}$, Carlos Felipe Grangeiro Loureiro ${ }^{2}$ e \\ Eiji Kawamoto ${ }^{3}$
}

\begin{abstract}
Resumo: Este trabalho tem como objetivos principais desenvolver e avaliar um algoritmo de médias sucessivas aplicado à reconstrução da matriz origem-destino (OD) sintética baseada em contagens de tráfego. Inicialmente, discute-se conceitualmente a estratégia de solução binível para este problema de otimização de ponto fixo e descreve-se a aplicação do método de médias sucessivas como técnica de solução hierárquica, detalhando o algoritmo proposto para implementação computacional. Em seguida, é apresentado um experimento para verificação do desempenho deste algoritmo, confrontando seus resultados com os provenientes do software TransCAD. As conclusões indicam que o método investigado foi capaz de gerar bons resultados, inclusive melhores que os do TransCAD. Entretanto, em nenhum dos casos analisados foi possível reconstruir a matriz original com elevada precisão. Ao final, são apontadas algumas questões de pesquisa que poderão contribuir para aplicações da modelagem da matriz OD sintética em redes congestionadas de transportes.
\end{abstract}

DOI:10.4237/transportes.v21i2.697.

Palavras-chave: Matriz OD Sintética; média Sucessiva; técnica bí-nível.

\begin{abstract}
This work has as its main objectives to develop and evaluate a successive averages algorithm applied to the reconstruction of the synthetic OD matrix based on traffic flow counts. Initially, it is discussed conceptually the bi-level solution strategy for this fixed-point optimization problem and described the application of the method of successive averages as a hierarchical solution technique, detailing the proposed algorithm for computer implementation. Following, it is presented an experiment to verify the performance of this algorithm, comparing its results to those obtained with TransCAD software. The conclusions indicate that the assessed method was able to produce good results, even better than TransCAD's. However, in none of the analyzed cases it was possible to reconstruct the original matrix with a high level of precision. At the end, some research questions are highlighted which may improve modeling efforts of the synthetic OD matrix in congested transportation networks.
\end{abstract}

Keywords: Synthetic OD matrix; successive average; bi-level method.

\section{INTRODUÇÃO}

O uso de modelos simplificados para a representação do padrão de deslocamentos em uma rede de transportes por meio de matrizes origem-destino (OD) vem sendo estudado há algumas décadas, com destaque para a modelagem da matriz OD sintética. Tal abordagem foi inicialmente proposta na década de 1970 para apoiar métodos expeditos de análise da demanda - em geral de curto prazo, já que não permite correlacionar a demanda com seus fatores causais e consiste na obtenção de uma matriz dos fluxos de viagens a partir da observação de volumes de tráfego (Willumsen, 1981). Tal simplificação pode ser interpretada como o inverso do problema da alocação do tráfego em uma rede de transportes, quando se busca reconstituir um conjunto de fluxos entre pares de zonas OD que, uma vez alocados na rede, reproduzam os volumes observados nos seus arcos. Conforme destacado por Hazelton (2001), nesse caso específico, o modelo sintético tem por objetivo reconstruir - e não estimar, como se convencionou chamar - uma matriz OD que resultou nos volumes observados em campo, consi-

\footnotetext{
${ }^{1}$ Bruno Vieira Bertoncini, Departamento de Engenharia de Transportes, Grupo de Pesquisa em Transporte, Trânsito e Meio Ambiente, Universidade Federal do Ceará, Fortaleza, CE, Brasil. (e-mail: bruviber@det.ufc.br).

${ }^{2}$ Carlos Felipe Grangeiro Loureiro, Departamento de Engenharia de Transportes, Grupo de Pesquisa em Transporte, Trânsito e Meio Ambiente, Universidade Federal do Ceará, Fortaleza, CE, Brasil. (e-mail: felipe@det.ufc.br).

${ }^{3}$ Eiji Kawamoto, Departamento de Engenharia de Transportes, Escola de Engenharia de São Carlos, Universidade de São Paulo, São Carlos, SP, Brasil. (e-mail: eiji@usp.br).
}

Manuscrito recebido em 18/5/2013 e aprovado para publicação em 15/7/2013. Este artigo é parte de TRANSPORTES v. 21, n. 2, 2013. ISSN: 2237-1346 (online). DOI:10.4237/transportes.v21i2.697. derando que a matriz obtida corresponde a apenas uma realização particular da população de matrizes que representam o fenômeno do padrão de deslocamentos na área, de forma que uma nova observação de volumes resultará na reconstrução de uma matriz OD diferente. Se, por outro lado, o objetivo for estimar os parâmetros populacionais do fenômeno em questão, tem-se então um problema distinto, adequadamente denominado de estimação de matrizes OD sintéticas (Pitombeira Neto et al., 2011).

Métodos de reconstrução de matrizes OD sintéticas, foco deste trabalho, ainda são pouco empregados pela comunidade científica e, principalmente, pela comunidade técnica, quando comparados com aplicações de modelos racionalistas (descritivos ou comportamentais) da demanda por viagens, tidos como "tradicionais" (Timms, 2001). Muito disto deve-se ao fato de que existem questões de pesquisa relativas tanto à formulação do problema da reconstrução da matriz OD sintética, quanto à sua solução, que ainda não foram suficientemente respondidas, gerando dúvidas quanto ao uso deste tipo de modelo. Nas últimas décadas, a comunidade internacional tem destinado esforços ao esclarecimento de questões relacionadas especialmente à solução deste problema, resultando em implementações de algoritmos distintos em pacotes comerciais de modelagem da demanda e simulação de redes de transportes, buscando a disseminação do uso deste método. Dentre as questões estudadas, destacam-se dois grupos: (i) aquelas relativas ao uso de informação adicional como, por exemplo, volumes de conversão observados nos nós (Bertoncini e Kawamoto, 2012) ou conhecimento a priori do padrão de deslocamento (Pitombeira Neto et al., 2011); e (ii) aquelas voltadas para o algoritmo de solução propriamente dito, com 
as propostas seminais de Robillard (1975) e Nguyen (1977) para redes viárias, respectivamente, sem e com congestionamento, contemplando uma solução hierárquica em dois níveis (alocação + reconstrução), ainda sendo utilizadas até hoje nos programas computacionais como base do método de reconstrução da matriz sintética.

O presente trabalho consiste em um esforço de pesquisa voltado aos algoritmos de solução, tendo sido motivado após testes com métodos implementados em pacotes comerciais QuEENSOD (Van Aerde, 1998) e TransCAD (Caliper, 2005) - que geraram resultados insatisfatórios, conforme avaliado nas dissertações de Bertoncini (2007) e Souza (2007), assim como na tese de Bertoncini (2010). Vale ressaltar que tais programas são do tipo "caixa preta", não permitindo acesso ao código implementado. Para melhor entender futuramente quais seriam as possíveis causas das falhas observadas, optouse neste trabalho por propor e implementar computacionalmente um método de solução também baseado na técnica binível, avaliando seu desempenho e estabelecendo as bases para uma posterior análise comparativa dos algoritmos implementados em alguns pacotes comerciais. Portanto, o objetivo geral deste trabalho é desenvolver e avaliar um algoritmo de médias sucessivas aplicado à reconstrução da matriz OD sintética. Seus objetivos específicos, com a correspondente organização do texto, são: (i) discutir conceitualmente a estratégia de solução bi-nível para o problema da reconstrução da matriz OD sintética (item 2); (ii) propor um algoritmo de médias sucessivas com uma estrutura adaptada de solução binível para este problema (item 3); (iii) verificar o desempenho da implementação computacional do algoritmo proposto por meio de um experimento controlado (item 4); (iv) analisar os resultados obtidos, confrontando-os com os provenientes do software TransCAD, considerado um dos programas mais conceituados para lidar com o problema da reconstrução (Timms, 2001) (item 5). Por fim, as conclusões da análise, com recomendação de questões pertinentes ainda a serem investigadas, são apresentadas no item 6.

\section{ESTRATÉGIA BI-NÍVEL NA RECONSTRUÇÃO DA MATRIZ OD SINTÉTICA}

A modelagem da matriz sintética tem por objetivo retornar a matriz OD que corresponda a um conjunto de observações de volumes de tráfego, ou, como colocado por Cascetta (1984), encontrar a matriz OD que minimiza uma medida de distância, "entrópica" ou euclidiana, em relação a uma matriz semente - gerada a partir de um modelo racionalista da demanda ou estimada para um período anterior - respeitando a restrição que, uma vez alocada na rede, reproduza os volumes observados em campo. Assim, a formulação do problema de modelagem da matriz OD sintética pode ser entendida como multicritério, tendo como objetivo minimizar duas funções de medidas de distância: uma relativa aos fluxos OD (demandas) e outra aos volumes de tráfego. Sua solução depende, portanto, da alocação de pesos a cada parcela da função objetivo. Se o analista acredita que o grau de confiabilidade dos fluxos OD é elevado, deve-se alocar um peso significativamente maior para a minimização das diferenças entre as demandas, aceitando-se soluções que incorporem erros na aproximação dos volumes. Caso contrário, as informações provenientes dos volumes observados em campo é que serão determinantes no processo. Esta análise pode ser traduzida matematicamente pela Equação (1), considerada uma equação geral para o problema da reconstrução (Cascetta, 2009):

$$
F=\underset{t \geq 0}{\arg \min }\left[\gamma_{1}\left(F_{1}(t, \hat{t})\right)+\gamma_{2}\left(F_{2}(v, \hat{v})\right)\right]
$$

Sujeito a:

$$
\sum_{i j} p_{i j}^{a} \cdot t_{i j}=v_{o b s}^{a}
$$

na qual, $F_{1}$ mede a distância entre a demanda modelada $(t)$ e a demanda conhecida a priori $(\hat{t})$ - também denominada matriz semente; $F_{2}$ mede a distância entre os volumes alocados $(v)$ e os volumes observados em campo $(\hat{v}) ; \gamma_{1}$ e $\gamma_{2}$ são parâmetros que refletem a incerteza contida em $t$ e $v$, respectivamente, correspondendo aos pesos alocados a cada parcela da função objetivo. Os valores assumidos por $\gamma_{1}$ e $\gamma_{2}$ irão depender da confiança que o modelador atribui a cada parcela, não havendo um valor padrão, pré-determinado, para os mesmos. A solução do problema representado por (1) é possível com uso de expedientes como maximização da entropia, minimização da informação, mínimos quadrados generalizados, dentre outros.

Inicialmente, modelos de reconstrução da matriz OD a partir de contagens de tráfego assumiam que as proporções de viagens de cada zona de origem $i$ para cada zona de destino $j$ usando um arco $a\left(p_{i j}{ }^{a}\right)$ seriam constantes e independentes do nível de congestionamento, correspondendo ao volume observado neste arco $\left(v_{o b s}{ }^{a}\right)$ conforme expresso na Equação (2), denominada "restrição-chave" do modelo (Willumsen, 1981).

Em redes não congestionadas, os caminhos escolhidos são, costumeiramente, aqueles de menor distância total, de forma que as proporções de viagens podem ser determinadas por um método tudo-ou-nada; ou seja, caso o arco $a$ pertença a rota escolhida, $p_{i j}=1$, do contrário será 0 . Em situações de congestionamento, por outro lado, as proporções variam de acordo com os níveis de carregamento da rede; isto é, dependem da estrutura e intensidade dos fluxos OD. Assim, os esforços de modelagem da matriz OD sintética e da alocação (escolha de rotas) do tráfego, essenciais para representar as relações entre demanda e oferta em redes de transporte, estão intimamente conectados (input de um é output do outro), podendo ser combinados em uma única formulação matemática com estrutura de solução hierárquica em dois níveis, conforme proposto originalmente por Robillard (1975) e Nguyen (1977).

Visando contribuir para uma melhor compreensão do grau de inter-relação entre esses dois problemas de redes de transportes, apresenta-se na Figura 1 um fluxograma detalhando a estrutura clássica de solução bi-nível adotada genericamente, na qual o problema da alocação é resolvido no $1^{\circ}$ nível, fornecendo as proporções dos fluxos OD em cada arco que alimentarão, juntamente com os volumes observados e uma matriz semente, a iteração seguinte do problema da reconstrução da matriz OD sintética, representado no $2^{\circ}$ nível. Essa estratégia bi-nível tem sido largamente empregada como método hierárquico de solução para o problema da matriz OD sintética, sendo que diferenças podem ocorrer no âmbito da técnica de alocação utilizada (tudo-ou-nada, equilíbrio determinístico e estocástico do usuário são as mais comuns) e no modelo de reconstrução adotado (os mais utilizados são os derivados dos conceitos de maximi- 
zação da entropia, minimização da informação e minimização das diferenças quadráticas). Tais diferenças conceituais e metodológicas podem resultar em soluções divergentes para um mesmo problema modelado, indicando que a estratégia empregada interfere diretamente nos valores dos fluxos "reconstruídos".

Seguindo a estrutura clássica de solução bi-nível representada na Figura 1, Cascetta e Postorino (2001) apresentaram ram uma proposta para obtenção da matriz OD sintética em que o primeiro nível é resolvido por método de ponto fixo, em particular pelo método de médias sucessivas. A partir da solução de equilíbrio gerada no primeiro nível, os autores reconstroem a matriz OD no segundo nível utilizando o método dos mínimos quadrados proposto por Cascetta (1984). Apesar desta ser considerada atualmente uma das soluções mais eficientes para o problema da reconstrução da matriz OD sintética, Yang et al. (1992) já alertavam que a premissa do equilíbrio na rede pode comprometer a qualidade da matriz obtida, especialmente no caso de análises operacionais baseadas em curtos períodos de observação.

\section{MÉTODO PROPOSTO DE RECONSTRUÇÃO POR MÉDIAS SUCESSIVAS}

Para a solução, também em estrutura bi-nível, do problema de reconstrução da matriz OD sintética em redes congestionadas - considerado um problema de otimização de ponto fixo assim como o problema da alocação por equilíbrio do usuário - é proposta uma adaptação do algoritmo de alocação por médias sucessivas, desenvolvido inicialmente por Powel e Sheffi (1982). Trata-se de um processo iterativo com convergência garantida, bastante eficiente e de fácil aplicação, que realiza a cada etapa uma alocação tudo-ounada das viagens entre cada par OD, permitindo que eventuais erros de carregamento sejam corrigidos ao longo das iterações, sem necessidade de conhecimento prévio das rotas no par OD (Sheffi, 1985).
O algoritmo proposto, denominado de Método de Reconstrução por Médias Sucessivas (MRMS), estabelece que, na etapa inicial $(k=1)$, os tempos de percurso em cada arco são calculados para condição de fluxo livre; tempos esses que permitem encontrar os caminhos mínimos entre $i j$. Para se obter a primeira matriz OD $\left(\mathrm{T}_{1}\right)$, propõe-se aqui a utilização do princípio da maximização da entropia, supondo que todas as viagens sejam feitas pelo caminho mínimo, isto é: se o arco $a$ integra o conjunto de caminhos de custo mínimo e os fluxos $t_{i j}$ utilizam esse arco, então $p_{i j}{ }^{a k}=1$, caso contrário $p_{i j}{ }^{a k}=0$. Este artifício faz com que a solução do primeiro nível não dependa da busca pelo equilíbrio, diferente do método apresentado por Cascetta e Postorino (2001). Vale ressaltar que a maximização da entropia, expressa pela Equação (3), consiste em uma proposta específica deste trabalho, podendo também ser utilizado qualquer outro expediente para a solução do problema de reconstrução.

$$
\text { Maximizar } \sum_{i, j}\left(-t_{i j} \cdot \ln t_{i j}+t_{i j}\right), \text { s.a. (2) }
$$

Na etapa seguinte $(k=2)$, devem ser calculados os tempos de viagem nos arcos da rede considerando os carregamentos da etapa anterior, obtendo-se assim a matriz auxiliar $\mathrm{T}_{2}$, também por maximização da entropia.

A partir das duas matrizes, $\mathrm{T}_{1}$ e $\mathrm{T}_{2}$, é obtida uma terceira matriz que corresponde à média ponderada com pesos (1$\left.\phi_{2}\right)$ e $\phi_{2}$, respectivamente. Esta média equivale a subtrair da matriz $\mathrm{T}_{1}$ uma parcela $\phi_{2} . \mathrm{T}_{1}$ e somar outra parcela $\phi_{2} . \mathrm{T}_{2}$. Ao reduzir a matriz $T_{1}$, indiretamente são reduzidos os fluxos resultantes nos caminhos mínimos da primeira etapa $(k=1)$, enquanto que ao somar a parcela $\phi_{2} . \mathrm{T}_{2}$ são adicionados fluxos nos caminhos mínimos da segunda etapa $(k=2)$; ou seja, de certa forma é transferida uma parte do fluxo $t_{i j}$ do caminho mínimo da primeira etapa para o caminho mínimo da segunda etapa.

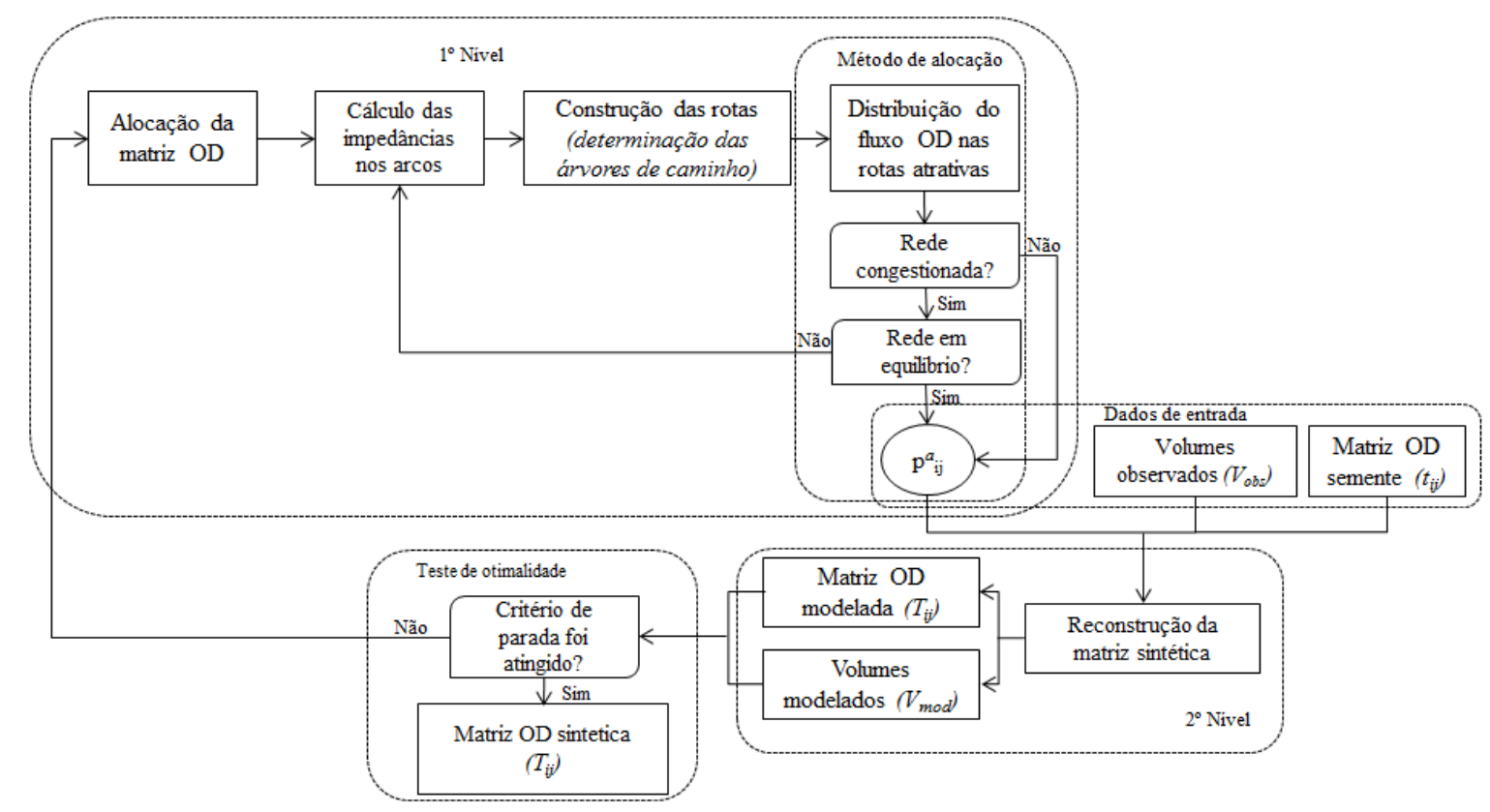

Figura 1. Estrutura bi-nível do processo de reconstrução da matriz OD sintética 
No caso de a rede não estar congestionada, o caminho mínimo da primeira etapa coincide com o caminho mínimo da segunda etapa e, portanto, a parcela de fluxo extraída de um caminho será devolvida ao mesmo caminho. Vale lembrar que nem sempre a parcela subtraída é igual à parcela adicionada, pois em redes congestionadas geralmente $\mathrm{T}_{1}$ é diferente de $\mathrm{T}_{2}$. Genericamente, na k-ésima etapa a subtração e o acréscimo podem ser feitos de acordo com o fator de ponderação $\phi_{k} \quad\left(0 \leq \phi_{k} \leq 1\right)$, calculado em função da etapa

(k) como segue:

$$
\phi_{k}=\frac{1}{k}
$$

Assim, a matriz OD média será definida como:

$$
\overline{\mathrm{T}}_{k}=\left(1-\phi_{k}\right) \cdot \overline{\mathrm{T}}_{k-1}+\phi_{k} \cdot \mathrm{T}_{k}
$$

em que $\overline{\mathrm{T}}_{\mathrm{k}}$ corresponde a matriz média de viagens estimada na etapa $k$. Adotando-se:

$$
\phi_{k}^{\prime}=\left(1-\phi_{k}\right)
$$

É possível expressar, de forma alternativa, a matriz média estimada na $k$-ésima etapa conforme a Equação (7). A matriz reconstruída na $k$-ésima iteração será dada como média aritmética das matrizes OD obtidas em cada uma das $k$ etapas consideradas.

$$
\overline{\mathrm{T}}_{k}=\frac{1}{k} \cdot \sum_{\mathrm{n}=1}^{k} \mathrm{~T}_{\mathrm{n}}
$$

O MRMS garante que as viagens resultantes em cada etapa $k$ utilizarão sempre os caminhos mínimos, distribuindo esse carregamento, em cada iteração, às demais rotas de acordo com uma taxa $\phi$, não necessitando conhecer previamente os caminhos alternativos. Conforme mencionado acima, o MRMS representa uma adaptação da estrutura clássica binível na qual não se faz necessário atingir o equilíbrio em cada iteração no primeiro nível. Além disso, a aplicação do método proposto constrói a solução com base apenas na parcela $F_{2}$ da Equação (1), ou seja, não requer informação adicional por meio de matriz prévia. $O$ fato de realizar a cada etapa uma alocação tudo-ou-nada se constitui em importante vantagem em termos de implementação computacional. Portanto, o MRMS busca reconstruir sinteticamente a matriz OD sem partir de uma matriz semente, seguindo uma adaptação da estratégia de solução em dois níveis na qual não se resolve o equilíbrio na rede em cada iteração, conforme descrito no fluxograma da Figura 2.

\section{TESTE DE VERIFICAÇÃO}

A verificação do desempenho do algoritmo MRMS proposto foi realizada utilizando um experimento com uma rede hipotética, pois seria inócuo desenvolver um experimento amparado em uma situação real que não permitiria inferir se possíveis erros seriam provenientes dos dados de entrada ou do método empregado. Assim, a rede viária analisada foi constituída de 9 nós (todos origem e destino) conectados entre si por 24 arcos orientados, conforme ilustrada na Figura 3. Essa rede hipotética foi carregada com uma matriz OD, denominada "real" (Tabela 1), objetivando: (i) fornecer os volumes de tráfego equivalentes a contagens in loco, obtidos a partir da alocação dos fluxos da matriz OD real à rede; e (ii) servir de referência na comparação dos resultados obtidos, permitindo assim mensurar os erros resultantes. Cada arco da rede adotada tem associado uma função matemática para cálculo do tempo de viagem, assumido como o único custo para utilização dos arcos, expressa conforme BPR (1964), com parâmetros $\alpha=0,15$ e $\beta=4$. Os valores do tempo de percurso em fluxo livre e da capacidade em cada arco encontram-se na Tabela 2.

A alocação da matriz OD à rede viária foi realizada utilizando o método do equilíbrio determinístico do usuário implementado no programa TransCAD 4.8, com seus resultados sendo verificados com base em uma rotina implementada em Microsoft Excel. O resultado da alocação é apontado ao lado dos respectivos arcos na Figura 3 como $V_{\text {obs }}$, uma vez que o volume resultante da alocação em um arco será considerado o volume observado naquele arco para efeito de reconstrução da matriz OD. Considerando que o número de incógnitas do problema corresponde à proporção de fluxo entre cada par OD alocado a uma das possíveis rotas que ligam este par OD, tem-se que o problema da reconstrução da matriz OD sintética que carrega a rede viária hipotética apresentada na Figura 3 possui no mínimo 72 incógnitas, caso apenas uma possibilidade de caminho entre cada par OD seja considerada, e no máximo 605 incógnitas, caso se considere todas as possibilidades de caminhos entre os pares OD (obtidos por enumeração exaustiva). Por outro lado, é possível obter no máximo 24 informações de volume provenientes das contagens nos arcos, considerando que na rede analisada não há arcos com volumes redundantes. Portanto, como o número de incógnitas é maior que o de informações, não há garantias de que a matriz reconstruída seja igual a real.

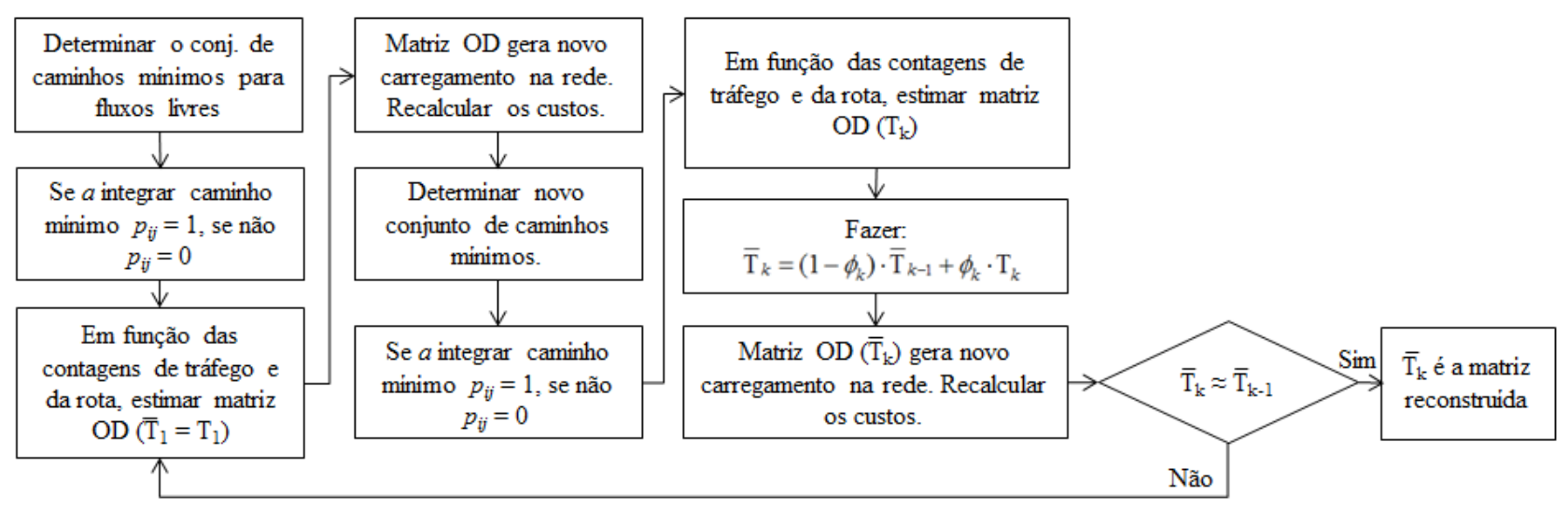

Figura 2. Algoritmo MRMS proposto 
Neste experimento, a reconstrução da matriz OD foi realizada utilizando dois métodos distintos. Primeiro, considerou-se a proposta do método MRMS, cujo algoritmo, descrito no item 3, foi implementado em uma planilha Microsoft Excel e teve o auxílio do suplemento Solver para atingir a solução. O segundo método escolhido, que permitiu confrontar o desempenho do MRMS, foi o implementado no TransCAD 4.8, pois trata-se de um método largamente utilizado e, assim como os disponíveis em outros pacotes comerciais, busca a solução de equilíbrio para o primeiro nível. $\mathrm{O}$ algoritmo implementado no TransCAD foi proposto por Nielsen (1993), sendo sensível à técnica de alocação e, principalmente, à qualidade da matriz semente. $\mathrm{O}$
TransCAD permite que o planejador escolha, dentre um conjunto de sete métodos de alocação - (i) não baseados em equilíbrio: tudo-ou-nada; incremental; restrição da capacidade; STOCH; (ii) baseados em equilíbrio: equilíbrio determinístico do usuário; equilíbrio estocástico do usuário; otimização do sistema - a técnica a ser empregada para solução do $1^{\circ}$ nível. Todavia, Nielsen (1998) faz a ressalva que seu modelo apresenta melhor desempenho quando utiliza a técnica de alocação baseada no equilíbrio determinístico do usuário. A solução para o $2^{\circ}$ nível procura minimizar a diferença entre o volume alocado e o volume observado na rede viária, fazendo com que a matriz reconstruída apresente estrutura semelhante à da matriz semente.

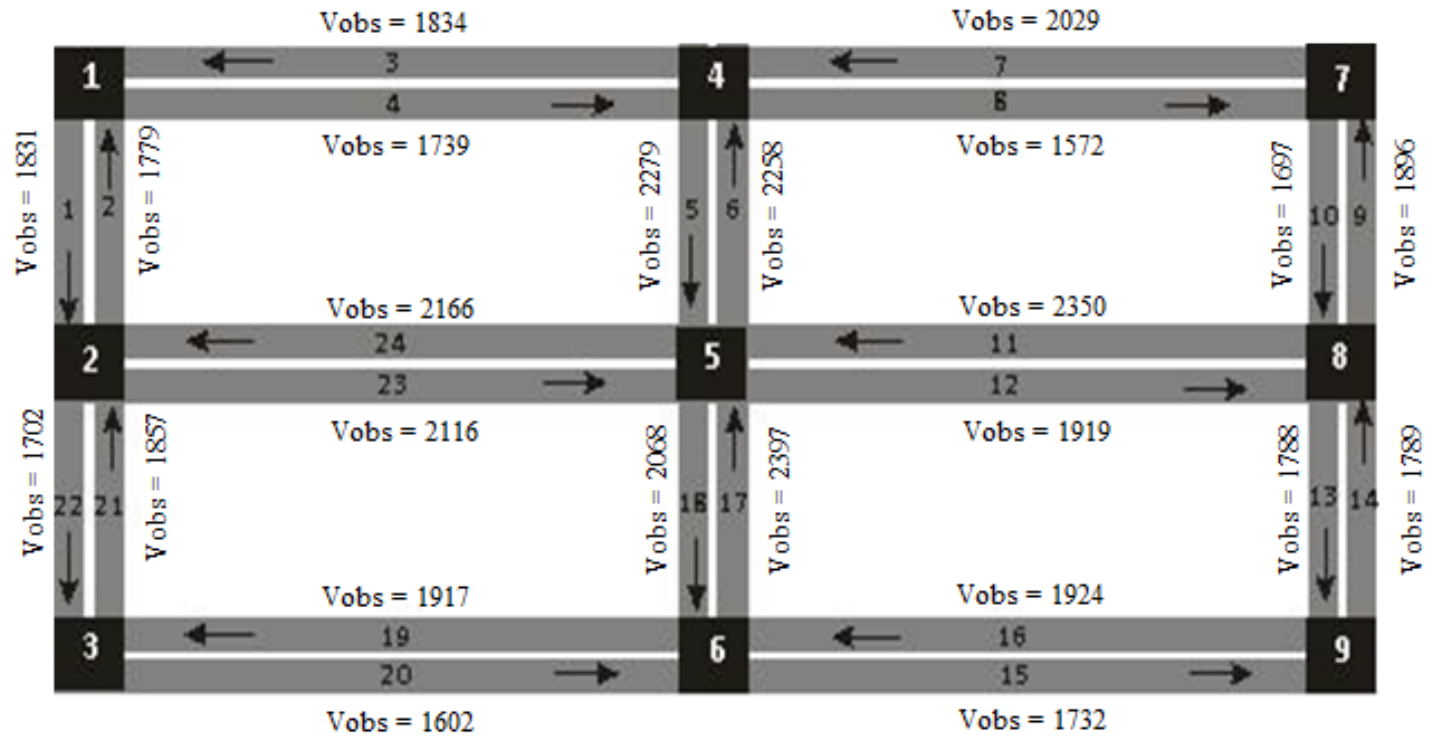

Figura 3. Rede viária hipotética

Tabela 1. Matriz OD "real"

\begin{tabular}{c|ccccccccccc}
\hline $\boldsymbol{O} \boldsymbol{D}$ & $\boldsymbol{1}$ & $\mathbf{2}$ & $\mathbf{3}$ & $\mathbf{4}$ & $\mathbf{5}$ & $\mathbf{6}$ & $\mathbf{7}$ & $\boldsymbol{8}$ & $\mathbf{9}$ & $\boldsymbol{\Sigma}$ \\
\hline $\mathbf{1}$ & - & 406 & 464 & 330 & 418 & 175 & 276 & 159 & 371 & $\mathbf{2 . 5 9 9}$ \\
$\mathbf{2}$ & 204 & - & 386 & 415 & 233 & 225 & 416 & 219 & 484 & $\mathbf{2 . 5 8 2}$ \\
$\mathbf{3}$ & 176 & 377 & - & 351 & 419 & 187 & 245 & 246 & 288 & $\mathbf{2 . 2 8 9}$ \\
$\mathbf{4}$ & 334 & 203 & 309 & - & 487 & 231 & 204 & 213 & 210 & $\mathbf{2 . 1 9 1}$ \\
$\mathbf{5}$ & 337 & 414 & 246 & 184 & - & 199 & 466 & 260 & 328 & $\mathbf{2 . 4 3 4}$ \\
$\mathbf{6}$ & 455 & 462 & 226 & 371 & 311 & - & 165 & 320 & 353 & $\mathbf{2 . 6 6 3}$ \\
$\mathbf{7}$ & 407 & 428 & 179 & 172 & 347 & 451 & - & 323 & 434 & $\mathbf{2 . 7 4 1}$ \\
$\mathbf{8}$ & 284 & 282 & 275 & 359 & 475 & 265 & 401 & - & 326 & $\mathbf{2 . 6 6 7}$ \\
$\mathbf{9}$ & 444 & 268 & 364 & 350 & 476 & 477 & 310 & 298 & - & $\mathbf{2 . 9 8 7}$ \\
\hline $\boldsymbol{\Sigma}$ & $\mathbf{2 . 6 4 1}$ & $\mathbf{2 . 8 4 0}$ & $\mathbf{2 . 4 4 9}$ & $\mathbf{2 . 5 3 2}$ & $\mathbf{3 . 1 6 6}$ & $\mathbf{2 . 2 1 0}$ & $\mathbf{2 . 4 8 3}$ & $\mathbf{2 . 0 3 8}$ & $\mathbf{2 . 7 9 4}$ & $\mathbf{2 3 . 1 5 3}$ \\
\hline
\end{tabular}

Tabela 2. Tempo de percurso na condição de fluxo livre e capacidade dos arcos da rede viária

\begin{tabular}{ccc|ccc}
\hline Arco & $\mathrm{T}_{0}$ & Capacidade & Arco & $\mathrm{T}_{0}$ & Capacidade \\
\hline 1 & 32 & 1520 & 13 & 32 & 1520 \\
2 & 32 & 1520 & 14 & 32 & 1520 \\
3 & 36 & 1610 & 15 & 36 & 1610 \\
4 & 36 & 1610 & 16 & 36 & 1610 \\
5 & 27 & 1650 & 17 & 27 & 1650 \\
6 & 27 & 1650 & 18 & 27 & 1650 \\
7 & 36 & 1610 & 19 & 36 & 1610 \\
8 & 36 & 1610 & 20 & 36 & 1610 \\
9 & 32 & 1520 & 21 & 32 & 1520 \\
10 & 32 & 1520 & 22 & 32 & 1520 \\
11 & 28 & 1530 & 23 & 28 & 1530 \\
12 & 28 & 1530 & 24 & 28 & 1530 \\
\hline
\end{tabular}


Portanto, diferentemente do MRMS, o método implementado no TransCAD depende do conhecimento prévio de uma matriz OD semente. Assim, a fim de realizar uma análise de sensibilidade dos impactos causados na reconstrução devido à qualidade da matriz semente, duas matrizes distintas representando situações extremas foram utilizadas: (i) matriz semente binária: equivalente à situação em que não é fornecida matriz semente, ou seja, todas as viagens têm a mesma probabilidade de ocorrer - neste caso, viagens intrazonais recebem valor 0 e as viagens interzonais recebem valor 1 , lembrando que o modelo sintético parte do pressuposto de que não existem viagens intrazonais (Willumsen, 1981); e (ii) matriz semente equivalente a $70 \%$ da matriz OD real (denominada S70): situação idealizada e bastante favorável, em que não há mudança no padrão de deslocamentos na área, mantendo-se a mesma estrutura da matriz OD, isto é, a probabilidade de ocorrer uma viagem de i para j, dada pela matriz OD semente, é igual à da matriz real. Os resultados provenientes do processo de reconstrução, assim como as respectivas análises, são apresentados e discutidos a seguir.

\section{ANÁLISE DOS RESULTADOS}

Uma primeira análise, feita a partir dos diagramas de dispersão dispostos na Figura 4, aponta que os valores resultantes do MRMS apresentam-se menos dispersos em relação aos valores reais que aqueles provenientes da reconstrução a partir do TransCAD; inclusive quando neste último foi utilizada matriz semente proporcional a matriz real $\left(\mathrm{S}_{70}\right)$. $\mathrm{O}$ valor do coeficiente de determinação $\left(\mathrm{R}^{2}\right)$ obtido com o método MRMS foi igual a $86 \%$, enquanto que para o TransCAD com matriz semente proporcional foi de $78 \%$. A diferença de resultados é mais evidente quando se observa o $\mathrm{R}^{2}$ proveniente dos resultados obtidos pelo TransCAD com uso de matriz semente unitária, que foi praticamente nulo.

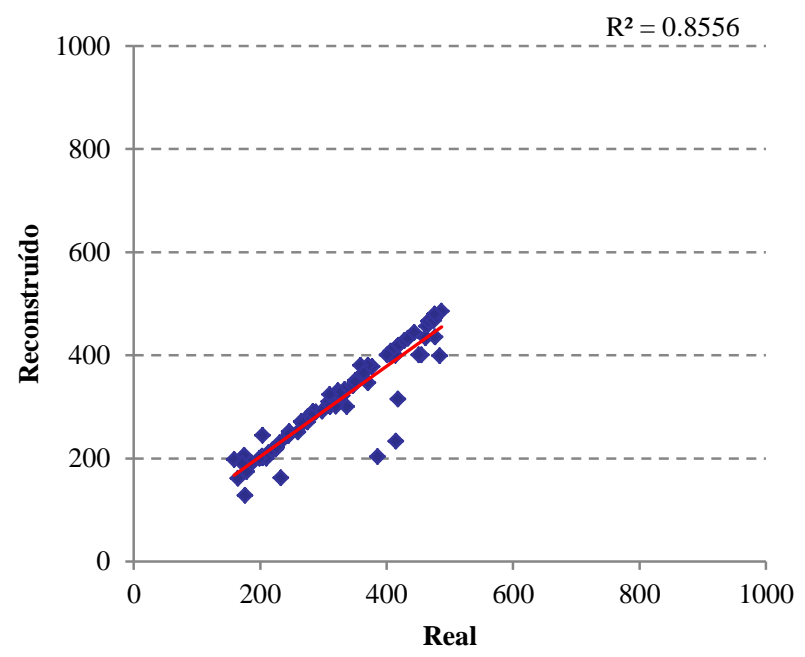

(a)

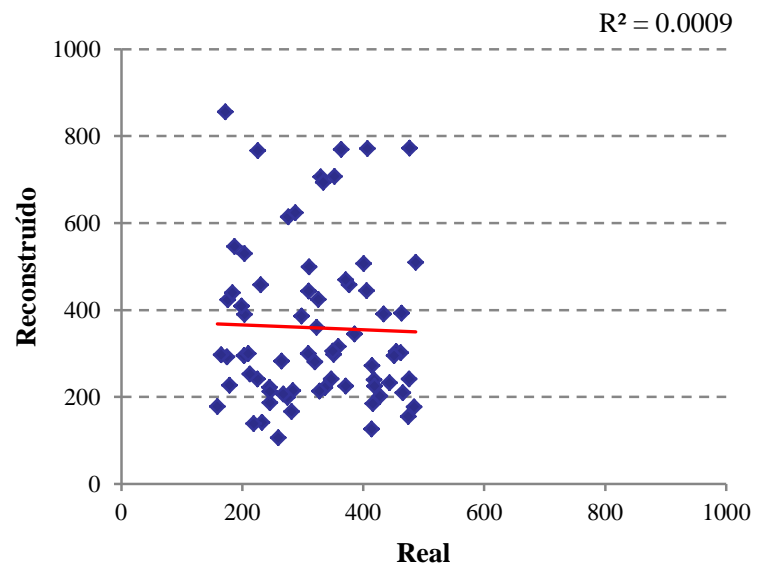

(b)

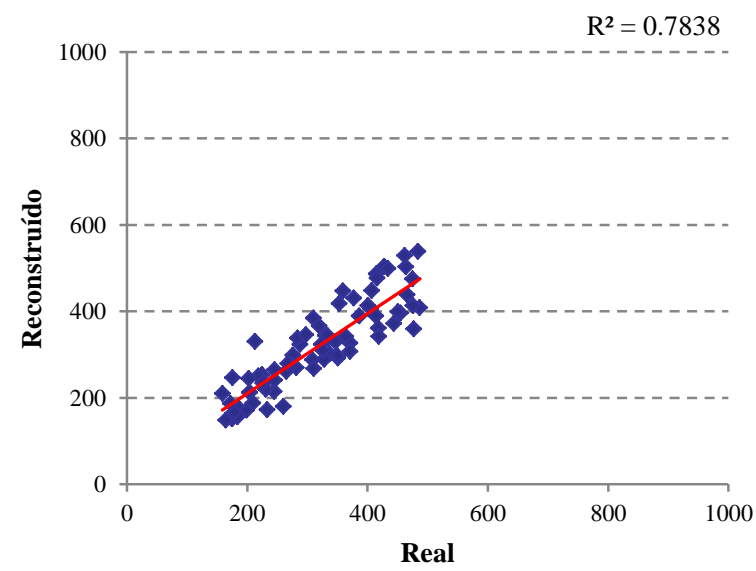

(c)

Nota: (a) resultados reais x reconstruídos usando o MRMS; (b) resultados reais x reconstruídos usando TransCAD com matriz semente unitária; (c) resultados reais x reconstruídos usando TransCAD com matriz semente proporcional a real

Figura 4. Diagrama de dispersão para os resultados obtidos. 
Outra análise foi realizada com base nas diferenças relativas entre os fluxos OD reais e reconstruídos, o que possibilitou verificar quanto o valor modelado está variando em relação ao real desagregadamente por par OD, permitindo uma melhor avaliação comparativa da eficiência dos métodos. A Figura 5 apresenta o histograma com as frequências simples absolutas das diferenças relativas, sendo possível observar que em 54 dos 72 pares OD a diferença relativa entre os resultados obtidos pelo MRMS e os fluxos reais foi no máximo de 5\%. Ao analisar os resultados do TransCAD com matriz semente proporcional à matriz real (TCDS70), somente 15 pares OD apresentaram erro relativo inferior a 5\%; já considerando o uso de matriz semente unitária (TCD), este número foi de apenas 2 pares, sendo que em outros 23 pares o valor do erro relativo foi superior a $60 \%$.

Analisando o gráfico (Figura 6) das distribuições acumuladas das diferenças relativas entre fluxos reais e reconstruídos pelos três métodos, pode-se constatar a superioridade do método MRMS em relação aos dois cenários considerados no TransCAD. O destaque negativo fica por conta do TransCAD quando faz uso de matriz semente unitária (TCD), pois o acúmulo de pares OD nas faixas menores de erro (até 30\%) foi muito pequeno, apenas $36 \%$ dos pares apresentaram erros desta magnitude, apontando para necessidade de uma investigação mais aprofundada sobre o método de reconstrução implementado no TransCAD, pois se trata de um dos principais métodos utilizados pela comunidade técnica e científica internacional.

\section{CONCLUSÕES E RECOMENDAÇÕES}

Os resultados obtidos no experimento com a rede viária hipotética reforçam a rejeição da hipótese teórica de reconstituição, com elevado grau de precisão, dos fluxos OD que originaram os volumes observados em campo, mesmo em uma situação representada por uma rede simples, com projeto viário homogêneo (arcos com capacidades e extensões bastante similares), contemplando uma matriz de viagens bastante equilibrada e volumes de tráfego consistentes em todos os seus arcos. Esta conclusão geral implica em uma recomendação à comunidade técnica de atenção às premissas e limitações dos métodos implementados em pacotes

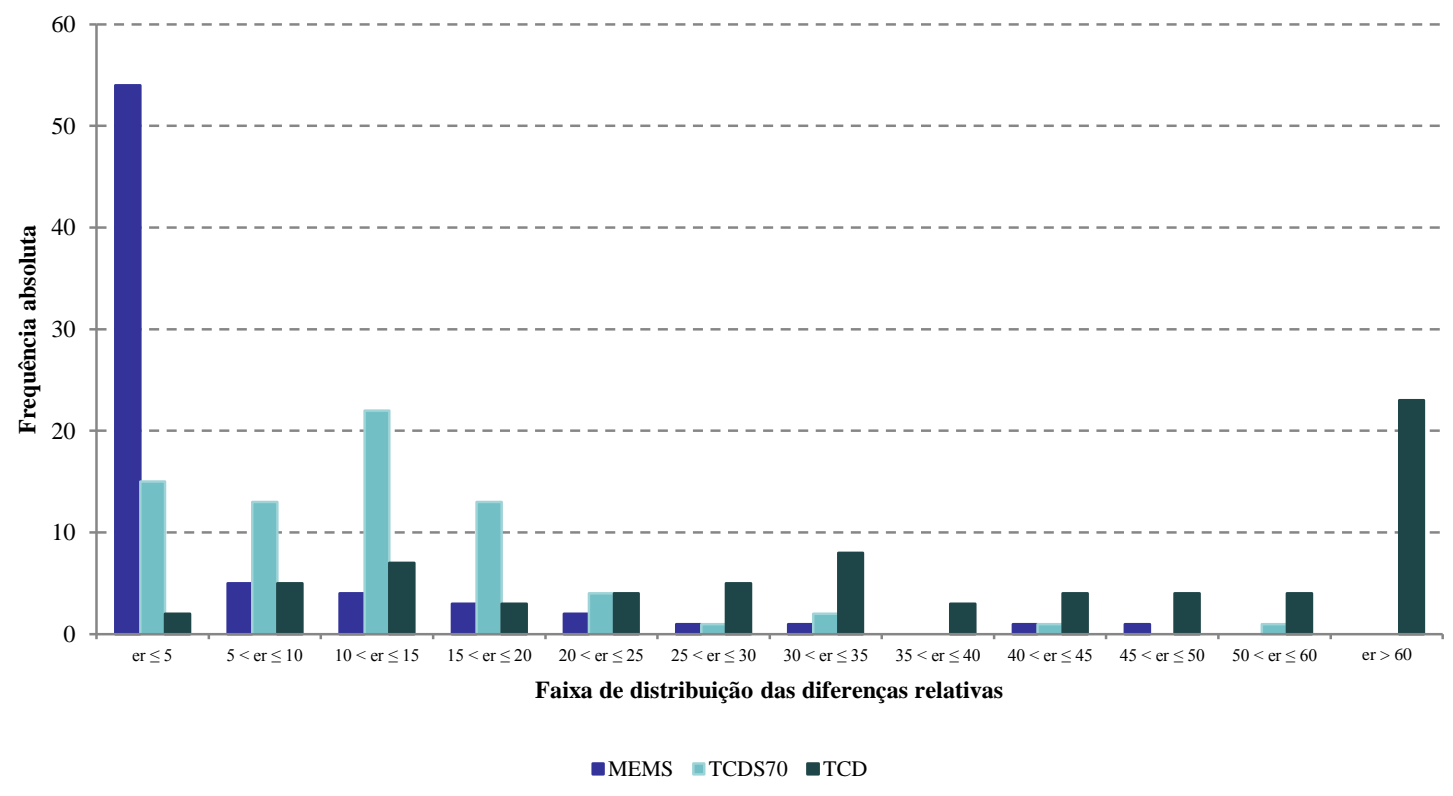

Figura 5. Histograma da distribuição absoluta das diferenças relativas entre viagens

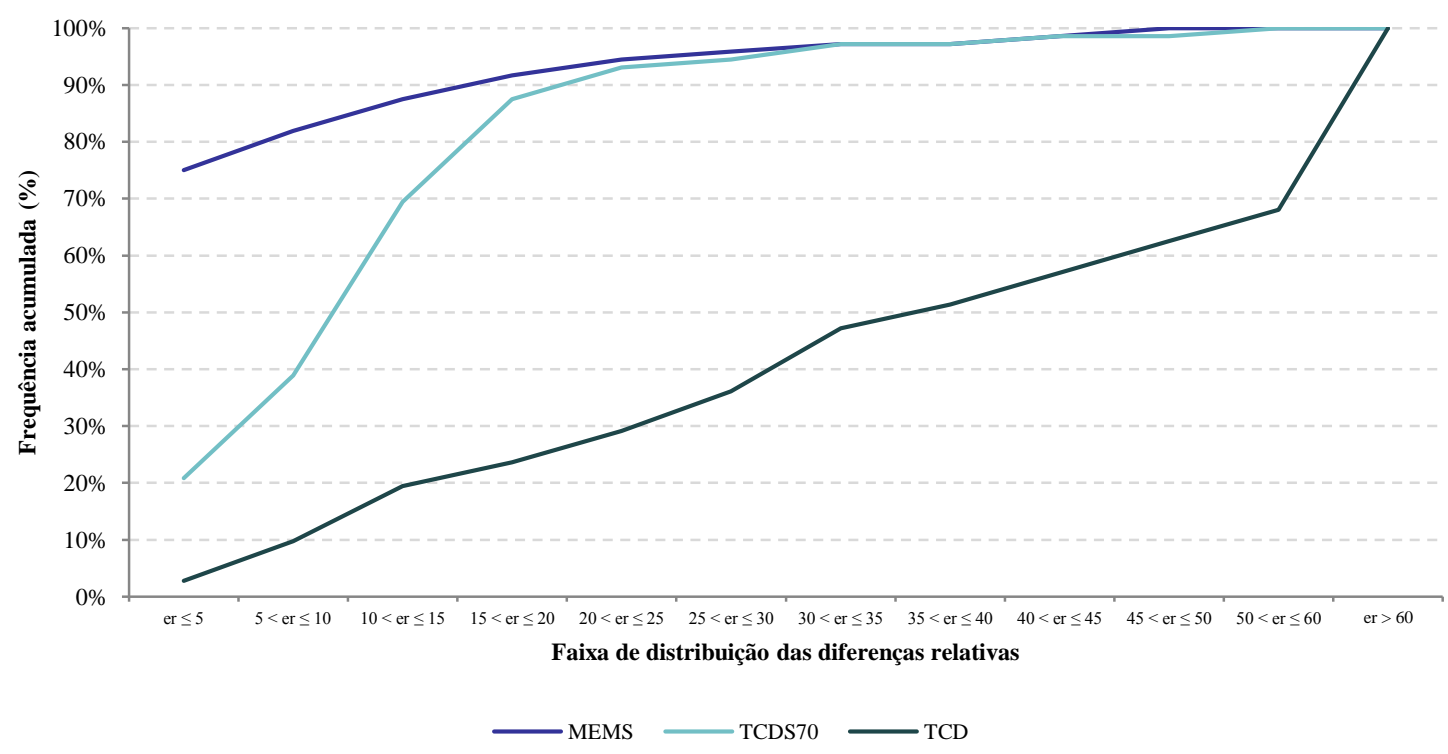

Figura 6. Histograma da distribuição acumulada das diferenças relativas entre viagens 
computacionais de modelagem de redes de transportes, especialmente em situações de baixa credibilidade quanto ao poder da matriz semente de representar o padrão de deslocamentos na área de estudo. Mesmo quando empregando algoritmos mais eficientes, devidamente validados pela comunidade científica, como aqueles baseados em métodos de otimização de ponto fixo, recomenda-se a realização de análises de sensibilidade quanto à quantidade e qualidade dos dados de volumes nos arcos, assim como quanto à crença no nível de representatividade da matriz semente. Esta recomendação decorre do fato de que, conforme ressaltado por Rakha et al. (2005), é teoricamente possível que existam múltiplas matrizes OD que repliquem exatamente os volumes observados em campo, levando à necessidade de avaliar qual delas seria a "mais provável" de ter gerado aquela configuração de carregamento da rede.

No que se refere especificamente ao método MRMS aqui proposto e avaliado, pode-se concluir que este foi capaz de reconstruir satisfatoriamente a matriz OD original da rede teste, sem a necessidade de conhecimento a priori do padrão de deslocamentos nessa rede. A matriz reconstruída pelo MRMS é determinada pela média aritmética das $k$ matrizes OD obtidas a partir de diferentes níveis de carregamento da rede, sendo este o diferencial do método. Vale ressaltar que o método proposto constrói a solução com base apenas na parcela $F_{2}$ da Equação (1), ou seja, não requer informação adicional por meio de matriz prévia, o que pode ser considerado uma vantagem significativa para a comunidade técnica, pois são raros os municípios brasileiros que dispõem deste tipo de informação. Ainda assim os resultados do MRMS obtidos no experimento foram superiores aos apresentados pelo TransCAD, mesmo quando este pacote foi alimentado por uma matriz semente proporcional à OD original, contexto este impossível de se reproduzir em redes reais.

Merece também destaque o fato de que a implementação do MRMS utilizada no experimento deste trabalho permitiu reconstruir a matriz OD sintética com base em uma estrutura de solução iterativa em dois níveis, considerando no primeiro nível o método de alocação tudo-ou-nada e no segundo o conceito de maximização da entropia, ou seja, a partir de procedimentos de fácil implementação computacional. Vale ainda ressaltar que o principal diferencial do método proposto reside na adaptação da estratégia clássica bi-nível de modo a não ser necessária a solução do equilíbrio na rede em cada iteração.

Como passos seguintes nesta linha de pesquisa sobre métodos de solução para o problema da reconstrução da matriz OD sintética em redes congestionadas, propõe-se uma análise de comparação do desempenho do MRMS com o método apresentado por Cascetta e Postorino (2001), realizando um experimento com a mesma rede teste utilizada pelos autores para verificar a eficiência da implementação computacional do seu algoritmo. O novo experimento deve possibilitar também a verificação do desempenho do MRMS em uma rede com maior quantidade de rotas entre pares OD, gerando diferentes condições de carregamento em situações nas quais não se tem contagens em todos os arcos. Nessa análise comparativa deve-se contemplar ainda a avaliação de variações do MRMS aqui descrito, incorporando outras técnicas de alocação, assim como princípios de otimização distintos do de maximização da entropia, permitindo uma melhor reflexão sobre a relevante questão de pesquisa inerente à validade da hipóte- se do equilíbrio estático em redes viárias urbanas, e a consequente necessidade de calibração do modelo de escolha de rotas para a reconstrução da matriz sintética.

Por fim, vale ressaltar a pertinência de se rever o papel dos modelos de reconstrução e estimação de matrizes OD sintéticas em análises estratégicas e operacionais de redes de transportes. Como argumentado neste trabalho, o conceito da reconstrução envolve a recuperação da matriz OD que gerou os dados de volumes observados. Em virtude do caráter estocástico dos fluxos nos pares OD, questiona-se a utilidade para o planejamento estratégico de se reconstruir uma matriz OD que representa somente uma realização dos fluxos em um dado instante ou período de tempo, em vez de estimar a tendência central e a dispersão do fenômeno do padrão de deslocamentos na área de estudo. Conforme já alertado por Timms (2001), a modelagem de reconstrução da matriz OD talvez seja mais apropriada a situações de gerenciamento do tráfego urbano em tempo real, nas quais se tem interesse na matriz OD "instantânea" referente ao curto período de tempo observado, enquanto modelos de estimação seriam mais eficientes nas aplicações de planejamento em que se tem interesse no comportamento médio dos fluxos OD em um período de tempo típico. Desta forma, espera-se com este esforço de pesquisa contribuir para uma melhor compreensão na comunidade técnico-científica das diversas premissas e formulações matemáticas da modelagem da matriz OD sintética, assim como das dificuldades práticas de implementação dos correspondentes algoritmos de solução, permitindo que técnicos e tomadores de decisão utilizem com mais segurança e propriedade este tipo de modelo nas análises de diagnóstico da problemática e avaliação de intervenções estratégicas e operacionais nos sistemas de transportes urbanos e regionais.

\section{AGRADECIMENTOS}

Os autores deste trabalho agradecem à FAPESP, CAPES e CNPq pelo financiamento da pesquisa. Ademais, os autores agradecem as relevantes sugestões e análises apresentadas pelos avaliadores deste artigo.

\section{REFERÊNCIAS BIBLIOGRÁFICAS}

Bertoncini, B. V. (2007) Uma proposta de carregamento incremental de fluxos veiculares para a estimação de matriz $O-D$ sintética. Dissertação (Mestrado). Escola de Engenharia de São Carlos, Universidade de São Paulo.

Bertoncini, B. V. (2010) Uma proposta de estimação de matriz OD a partir dos fluxos de tráfego observados nas interseções da rede de transportes. Tese (Doutorado). Escola de Engenharia de São Carlos, USP.

Bertoncini, B. V. e E. Kawamoto (2012) Modelagem da matriz OD sintética a partir dos volumes observados nas interseções da rede de transportes. Transportes, v. 20, n. 2, pp. 75-83. DOI:10.4237/transportes. v20i2.562.

Bureau of Public Roads - BPR (1964) Traffic Assignment Manual, Washington, DC, USA

Caliper (2005) Travel Demand Modeling with TransCAD 4.8. Caliper Corporation, Newton, USA.

Cascetta, E. (1984) Estimation of Trip Matrices from Traffic Counts and Survey Data: a Generalized Least Squares Estimator. Transportation Research Part B, v. 18, n. 4-5, p. 289-299. DOI:10.1016/01912615(84)90012-2.

Cascetta, E. e M. N. Postorino (2001) Fixed Points Approches to the Estimation of O/D Matrices Using Traffic Counts on Congested Networks. Transportation Science, v. 35, n. 2, p. 134-147. DOI:10.1287/ trsc.35.2. 134.10138.

Cascetta, E. (2009) Transportation Systems Analysis: Models and Applications (2a. ed.). Springer. DOI:10.1007/978-0-387-75857-2.

Hazelton, M. L. (2001) Inference for origin-destination matrices: estimation, 
prediction and reconstruction. Transportation Research B, v. 35, n. 7, p.667-676. DOI:10.1016/SO191-2615(00)00009-6.

Nguyen, S. (1977) Estimating an OD matrix from network data: a network equilibrium approach. Publication 87. Centre de Recherche sur les Transports, Université de Montreal.

Nielsen, O. A. (1998) Two New Methods for Estimating Trip Matrices from Traffic Counts. Travel Behaviour Research: Updating the State of Play. Ed. Elsevier.

Pitombeira Neto, A. R.; B. V. Bertoncini e C. F. G. Loureiro (2011) Abordagem bayesiana na estimação de matrizes origem-destino sintéticas em redes de transportes. In: Anais do XXV ANPET - Congresso de Pesquisa e Ensino em Transportes, Belo Horizonte.

Powell, W. B. e Y. Sheffi (1982) The convergence of equilibrium algorithms with predetermined step sizes. Transportation Science, v. 16, n. 1, p. 45-55. DOI: 10.1287/trsc.16.1.45.

Rakha, H.; H. Paramahamsan; M. Van Aerde (2005). Comparison of static maximum likelihood Origin-Destination formulations. Institute for Operations Research and the Management Sciences.

Robillard, P. (1975) Estimating the O-D matrix from observed link volumes. Transportation Research Part B, n. 9, p. 123-128.

Souza, D. D. M. R. (2007) Estimação Sintética de Matrizes Origem/Destino a partir de Contagens Volumétricas em Áreas com Controle do Tráfego em Tempo Real com o Auxílio do Queensod. Dissertação (Mestrado). Programa de Mestrado em Engenharia de Transportes, Universidade Federal do Ceará.

Timms, P. (2001) A philosophical context for methods to estimate origindestination trip matrices using link counts. Transport Reviews, v. 21, n. 3, p. 269-301. DOI:10.1080/713868144.

Van Aerde, M. (1998) QueensOD - Release 2.10 - User's Guide: estimating origin destination traffic demands from link flow counts. Michel Van Aerde \& Associates, Ltd, Blacksburg, VA, USA.

Sheffi, Y. (1985) Urban transportation networks: Equilibrium analysis with mathematical programming methods. Prentice Hall, Englewood Cliffs, NJ, p 367-374

Willumsen, L.G. (1981) Simplified transport models based on traffic counts. Transportation, n. 10, p. 257-278. DOI:10.1007/BF00148462.

Yang, H.; T. Sasaki; Y. Iida e Y. Asakura (1992). Estimation of OriginDestination Matrices from Link Traffic Counts on Congested Networks. Transportation Research Part B, v. 26, n. 6, p. 417-434. DOI:10.1016/ 0191-2615(92)90008-K. 